\title{
Enhancing GMI properties of melt-extracted Co-based amorphous wires by twin-zone Joule annealing
}

\author{
J.S. Liu ${ }^{\text {a }}$, F.Y. Cao ${ }^{\text {a }}$, D.W. Xing ${ }^{\text {a }}$, L.Y. Zhang ${ }^{\text {a }}$, F.X. Qin ${ }^{b}$, H.X. Peng ${ }^{\text {b,c }}$, X. Xue ${ }^{\text {a }}$, J.F. Sun ${ }^{\text {a,* }}$ \\ ${ }^{\mathrm{a}}$ School of Materials Science and Engineering, Harbin Institute of Technology, Harbin 150001, China \\ ${ }^{\mathrm{b}}$ Advanced Composite Center for Innovation and Science (ACCIS), Department of Aerospace \\ Engineering, University of Bristol, University Walk, Bristol BS8 1TR, United Kingdom \\ ${ }^{\mathrm{c} C e n t r e ~ f o r ~ N a n o s c i e n c e ~ a n d ~ Q u a n t u m ~ I n f o r m a t i o n, ~ U n i v e r s i t y ~ o f ~ B r i s t o l, ~ T y n d a l l ~ A v e n u e, ~ B r i s t o l ~ B S 8 ~}$ \\ 1FD. United Kingdom
}

\section{ABSTRACT}

The influence of twin-zone Joule annealing (TJA) on the microstructure and magnetic properties of melt-extracted $\mathrm{Co}_{68.2} \mathrm{Fe}_{4.3} \mathrm{~B}_{15} \mathrm{Si}_{12.5}$ amorphous microwires has been investigated. Experimental results indicated that twin-zone Joule annealing treatment improved the GMI property of as-cast wires to a greater extent comparing with Joule annealing (JA) and conventional vacuum annealing (CVA) techniques. At $15 \mathrm{MHz}$, e.g., the maximum GMI ratio $\left[\Delta Z / Z_{0}\right]_{\max }$ of a TJA wire increases to $104.29 \%$, which is more than 5 times of $20.49 \%$ for the as-cast wire, nearly 2 times of $56.47 \%$ for the JA wire, while the CVA wire has a decreased GMI ratio; the field response sensitivity of the TJA wire increased to $171.62 \% / \mathrm{Oe}$ from $80.32 \% / \mathrm{Oe}$ for the as-cast wire, exceeding the values of $140.76 \% / \mathrm{Oe}$ for the JA wire and of $39.17 \% / \mathrm{Oe}$ for the CVA wire. The stress or structural relaxation in TJA wire increases circumferential permeability, and magnetic moment achieves a critical state of excitation for overcoming eddy-current damping or "nail-sticked" action in rotational magnetization process at relatively high frequency. From the mictrostructural point of view, the role of regularly arranged atomic micro-regions (RAAM) and of medium range order region (MROR) determines the efficiency of various annealing techniques. Conclusively, TJA is established as an efficient annealing technique to enhance the GMI effect of wires, which can subsequently be used for detecting weak magnetic field at relatively wider working range $( \pm 2.0 \mathrm{Oe})$.

Keywords: twin-zone Joule annealing (TJA); melt-extracted amorphous wires; microstructure; GMI effect;

* Corresponding author at: School of Materials Science and Engineering, Harbin Institute of Technology, No.92 West Da Zhi St., Harbin, Heilongjiang, China. Tel.: +86-451-86418317; Fax: +86-451-86413904. E-mail address: jfsun_hit@263.net (J.F. Sun). 
sensor application

\section{Introduction}

Giant magnetoimpedance (GMI) amorphous microwires have been of much interest due to their high-performance applications as sensor elements particular to weak magnetic field detection [1-4]. Albeit the flourish of a variety of preparation techniques, improvement of existent techniques and development of new ones remain to be explored [5]. Melt-extraction, for one, has been recently revisited with a new technical update [6], which satisfactorily yields high-quality microwires with superior soft magnetic properties when compared with other approaches such as in-rotating water spinning, Taylor-wire and glass-coated melt spinning. The advantages of melt-extraction consist in: (i) it has the highest solidification or cooling rate, which enables the wires to possess an excellent soft magnetic property; (ii) the melt-extracted wires without glass cover are more suitable for electronic package and sensor applications; (iii) the parameters (i.e., linear velocity of wheel, feed rate of the molten) of melt-extraction can be conveniently controlled to produce wires with uniform diameter and roundness [7-10].

In another perspective, post-processing treatments are duly developed in order to further improve the performance of wires for specific applications [11-13]. C. Morón et al. [14] found that CVA Fe-based nanocrystalline wire enhanced magnetic properties to a limited extent, such as GMI ratio increased from $1 \%$ of as-cast to 5\% after CVA. V. Zhukova et al. [15] reported that dc current annealing (CA) with or without dc axial magnetic field can significantly affect magnetic properties of glass-coated microwires, and the noticeable longitudinal magnetic anisotropy induced by the combined effects of the magnetic field and internal stresses arising from the coating during the thermal treatment. K.R. Pirota et al. [16] observed that Joule annealing led to a short-range order relaxation and hence greatly improved the soft magnetic properties of Co-based glass-coated wire, with the GMI ratio 
$\left[\Delta \mathrm{Z} / \mathrm{Z}_{\max }\right]_{\max }$ at $15 \mathrm{MHz}$ of about $600 \%$ for special annealing conditions (10 min annealing with applied current of 70mA). J.M. Blanco et al. [17] also observed a GMI ratio $\left[\Delta Z / Z_{\max }\right]_{\max }$ of up to $330 \%$ for Co-based amorphous wire treated by Joule heating under applied torsion which exhibits a tendency to achieve a slightly asymmetric character at certain torsion. It follows these previous work that the soft magnetic property and GMI effect of wires can be effectively improved by various annealing techniques, which can be explained by the mechanisms of induced strong circumferential anisotropy and suitable stress releasing. As long as the annealing process is under delicate control to make the better of these two mechanisms, there would still be room to further improve GMI effect of microwires. Herein, a novel annealing technique, twin-zone Joule annealing (TJA), is proposed in the present study to seek for a stronger GMI effect.

In theory, comparing with CVA and JA, TJA could not only effectively avoid the crystallization and surface oxidation of wire resulting from high temperature during CVA but also release the inner residual stress adequately and improve structural relaxation, to further condition the original domain structure and magnetic properties. From the fundamental structure-property point of view, their magnetic properties are closely related to microstructures yielded by various annealing [18]. For instance, the formation of nanophases can be used to improve the soft magnetic properties of Co-based amorphous alloy and amorphous ribbons, but excessive amount of nanophases could deteriorate soft magnetic properties with increasing processed temperature $[19,20]$. However, only a few researchers resorted to high-resolution transmission electron microscopy (HRTEM) to explore the detailed information of microstructure, which otherwise would be instructive to all relevant studies: this will free the explanation of how annealing techniques modify GMI from speculations and a better understanding of this matter is likely to lead to development of new techniques. Therefore, it is worthwhile to conduct a detailed study on the relationship between 
microstructures and magnetic properties with the aid of HRTEM.

In this context, we tend to approach a somewhat old topic from new perspectives, i.e., an updated fabrication technique, a new annealing method, and a new characterization approach. The proposed TJA technique will be evaluated in terms of its effectiveness on the GMI enhancement of as-cast wires in comparison with conventional techniques with reference to microstructure information provided by HRTEM.

\section{Experiments}

The mother alloy ingot with nominal composition of $\mathrm{Co}_{68.2} \mathrm{Fe}_{4.3} \mathrm{~B}_{15} \mathrm{Si}_{12.5}$ (in at. \%) for meltextraction was prepared by arc-melting in pure argon and copper mould casting methods. In melt-extraction facility, the starting alloy in $\mathrm{BN}$ crucible was melted by induction coil. Subsequently, the microwires with diameter ranging from $40 \mu \mathrm{m}$ to $80 \mu \mathrm{m}$ were extracted by the edge of a high speed rotating copper wheel in purified argon atmosphere. Conventional vacuum annealing (CVA) with single isothermal annealing stage was carried out in a tubular furnace with $10^{-3}$ torr vacuum at $773 \mathrm{~K}$ (between crystallization temperature $T_{\mathrm{x}}=785.6 \mathrm{~K}$ and Curie temperature $T_{\mathrm{c}}=675.0 \mathrm{~K}$ ) for $20 \mathrm{~min}$ and then the wires were naturally cooled in the furnace. Joule annealing (JA) was conducted under a current intensity of $100 \mathrm{~mA}\left(4.21 \times 10^{5}\right.$ $\mathrm{A} / \mathrm{dm}^{2}$ ) for $20 \mathrm{~min}$ and then the wires were naturally cooled in air. Moreover, the proposed twin-zone Joule annealing (TJA) consists of two successive stages: one annealing stage with duration $15 \mathrm{~min}$ at current intensity of $80 \mathrm{~mA}\left(3.37 \times 10^{5} \mathrm{~A} / \mathrm{dm}^{2}\right)$ and another annealing stage with duration $5 \mathrm{~min}$ at current intensity of $110 \mathrm{~mA}\left(4.63 \times 10^{5} \mathrm{~A} / \mathrm{dm}^{2}\right)$. The former stage could release internal stress adequately, the latter stage can generate toroidal magnetic field by DC current to induce structural relaxation with circumferential magnetic moments in the wires.

As-cast and annealed wires were examined by $\mathrm{X}$-ray diffraction with $\mathrm{CuK}_{\alpha}$ radiation $(\mathrm{XRD}$, Rigaku D/max- $\gamma \mathrm{B}$ ), high-resolution transmission electron microscopy (HRTEM, JEM 2010F). The HRTEM sample was prepared by using a Gatan 691 ion beam thinner, the corresponding 
process depicted schematically in Fig. 1 is detailed as follows: firstly, the wires with different lengths were arranged onto a copper ring with a diameter of $3 \mathrm{~mm}$ using glue-bond to prevent the wires from separation, and then another copper ring with glue-layer was used to M-bond the previous ring together; subsequently, the sample with the double copper rings was heated in the water-bath for $10 \mathrm{~min}$ for glue solidification; finally, the sample was thinned by ion beam thinner for 3-5 hours at a voltage of $3.5 \mathrm{kV}$ until the wedged sample thickness was reduced to about one nanometer, where the upper and lower ion beams are with a tilt angle of $7^{\circ}$ and $8^{\circ}$, respectively.

All samples for impedance measurement are about $55 \mu \mathrm{m}$ in diameter and $12 \mathrm{~mm}$ long. The impedance measurements were performed using Aligent 4294A precision impedance analyzer at frequencies $40 \mathrm{~Hz}-110 \mathrm{MHz}$. The GMI ratio, $\Delta \mathrm{Z} / \mathrm{Z}_{0}$, is defined as [21]:

$$
\frac{\Delta \mathrm{Z}}{\mathrm{Z}_{0}}(\%)=\left[\frac{Z\left(H_{e x}\right)-Z\left(H_{0}\right)}{Z\left(H_{0}\right)}\right] \times 100 \%
$$

and the magnetic field response sensitivity of GMI is expressed as [22]:

$$
\xi=\frac{d\left[\frac{\Delta Z}{Z_{0}}(\%)\right]}{d H_{e x}}
$$

where $\mathrm{Z}\left(H_{\mathrm{ex}}\right)$ is the impedance under external field, $H_{\mathrm{ex}}(\leq 4.2 \mathrm{Oe})$, applied by a solenoid. $\mathrm{Z}\left(H_{0}\right)$ is the initial impedance at $0 \mathrm{Oe}$. All measurements were performed at room temperature.

\section{Results and discussions}

\subsection{Microstructural evolution during annealing treatment}

Fig. 2 shows that the X-ray diffraction patterns of the as-cast wire, either JA or TJA wires contains one broad diffuse peak, which is a representative characteristic for amorphous phase and indicates the absence of obvious crystalline phase in the wires. Whereas, several specific and somewhat weak diffraction peaks appear in the diffraction pattern of the wire after CVA treatment at $773 \mathrm{~K}$ for $20 \mathrm{~min}$. These peaks correspond to the hard magnetic phases CoSi along crystal plane (210), implying that the CVA treatment has induced the formation of crystalline 
phases in the wire.

It is well known that the valid volume fraction for X-ray diffraction for a mixed sample is limited to a lower boundary of $2 \%$, which means if the volumes of the crystalline phases in the as-cast, JA and TJA wires are lower than $2 \%$ of the sample's volume they could not be detected by X-ray. HRTEM analysis is then employed here to confirm their microstructure. Fig. 3 displays the HRTEM images and patterns, including Fourier Function Transform (FFT) and Inverse Fourier Function Transform (IFFT) micrographs, and partial magnifications of as-cast and different annealed wires. HRTEM image (Fig. 3 (a)) of the as-cast wire does not exhibit any crystalline region, corresponding electron diffraction pattern is diffuse halo ring and the IFFT image including its partial magnifications ( I and II) demonstrates fully disordered atomic arrangement, i.e., amorphous state. Different from the IFFT image of ascast wire, the IFFT images of JA and TJA wires (as shown in Fig. 3 (b) and Fig. 3 (c), respectively) display several regularly arranged atomic micro-regions (RAAM) shown as cystiform shape and strip shape with atoms planes (III-VI). The RAAMs in the IFFT image of TJA wires are larger in size than those of JA wires. It can be concluded that the extent of atomic diffusion and rearrangement in the TJA wires is higher than that of JA wires. Fig. 3 (d) demonstrates that the HRTEM image of CVA wire contains a large number of regions with atomic planes structure conspicuously shown in the IFFT image, where the strip shape RAAM not only bears larger size and volume fraction than that of JA and TJA wires but also shows more regularly arranged atomic planes (VII and VIII).

The size of the RAAMs shown in Fig. 3 (b) - (d), is in the range of about 1.0nm-3.0nm, which falls in the range of $0.5 \mathrm{~nm}-3.0 \mathrm{~nm}$ for atomic configuration considered as medium range order region (MROR) [23]. Therefore, annealing treatment induces MRORs in the amorphous wires and consequently increases the regularity of atomic arrangement. The formation mechanism of the MRORs during the annealing treatment can be reasoned as 
follows. Rapid freezing causes the as-cast wire to keep the short range order and long range disorder, i.e. the amorphous microstructure, and also generates residual internal stress and local magnetic anisotropy field in the wire. During annealing, all the atoms will diffuse from high chemical potential region to low chemical potential region as a result of thermal activation, and atoms with large chemical affinity tend to form stable phase. All these effects can be enhanced with increasing annealing temperature (below Curie temperature). For CoFeBSi system, cobalt atoms have affinity to form clusters in a random network, and further associate with silicon atoms to form the CoSi cluster or even CoSi crystalline phase with larger size. The average temperatures of JA and TJA state microwires during annealing can be evaluated using the energy conservation law as follows [24]:

$$
\left\{\begin{array}{l}
\rho_{M} c \frac{d T}{d t}-Q_{E}-Q_{h}-Q_{r}=0 \\
Q_{E}=I^{2} R \\
Q_{h}=h_{\mathrm{c}} S\left(T_{A i r}-T\right) \\
Q_{r}=\varepsilon_{w} \sigma_{s b} S\left(T_{A i r}{ }^{4}-T^{4}\right) \\
\Delta T=\frac{\left(Q_{E}+Q_{h}+Q_{r}\right) \cdot \Delta t}{\rho_{M} c}
\end{array}\right.
$$

where $\rho_{M}$ is the mass density of wire, $7720 \mathrm{~kg} / \mathrm{m}^{3} ; c$ is the specific heat, $463.18 \mathrm{~J} \cdot(\mathrm{kg} \cdot \mathrm{K})^{-1}$; $Q_{E}$ is the Joule heat during the current passing through the wire, $I$ is the dc current density; $Q_{h}$ is the natural-convection heat transfer, $h_{\mathrm{c}}$ is the coefficient of heat transfer, $1.02 ; Q_{r}$ is the radiative heat, $\varepsilon_{\mathrm{W}}$ is the coefficient of thermal emittance, $0.43 ; S$ is the surface area of wire, $S=2 \pi a \cdot l, a, l$ are the radius and length of microwire, respectively; $\sigma_{\mathrm{sb}}$ is the Stefan Boltzmann constant, $\sigma_{\mathrm{sb}}=5.67 \times 10^{-8} \mathrm{~W} / \mathrm{m}^{2} \cdot \mathrm{K}$. Therefore, the calculated average temperatures $T_{c a}$ of JA and TJA processes are around $428.4 \mathrm{~K}$ and $554.7 \mathrm{~K}$, respectively; both are much lower than annealing temperature of CVA, $T_{\mathrm{a}}=773.0 \mathrm{~K}, T_{\mathrm{x}}=785.6 \mathrm{~K}$ and $T_{\mathrm{c}}=675.0 \mathrm{~K}$. It can 
be concluded that the size and volume fraction of MRORs increase with annealing temperature, especially when $T_{\mathrm{a}}$ is close to the $T_{\mathrm{x}}$. Therefore, the CVA wire has more MRORs than JA and TJA wires, and MRORs can significantly influence the domain wall movement, magnetic moment rotation and magnetic properties. The TJA wire exhibits higher $T_{\mathrm{ca}}$ than JA wire, and its inner atoms have stronger diffusion ability to form RAAM under the effects of inner residual stress relief and relatively high annealing current. Therefore, the TJA wires are all the more likely to possess better soft magnetic properties than CVA and JA wires.

\subsection{Effect of various annealing treatments on GMI properties}

In general, most suitable annealing treatments are capable of improving GMI effect of as-cast wires. It has been reported previously, for example, that Joule annealing (JA) is an effective way of generating circular magnetic field induced by the electric current and hence circumferential magnetic anisotropy for a wire-shaped sample to enhance its magnetoimpedance ratio, and that JA works better than CVA $[11,12,25]$. Thus, it is necessary to conduct a comparative study to identify the uniqueness of TJA method.

Fig. 4 shows the GMI ratio $\Delta \mathrm{Z} / \mathrm{Z}_{0}$ profiles of as-cast and different annealed states at four different frequencies. With an increase of the DC magnetic fields, all curves display good symmetry. Notably, at low frequency of $100 \mathrm{kHz}$ (Fig. 4 (a)), the $\Delta \mathrm{Z} / \mathrm{Z}_{0}$ curve has a single peak (SP) appearing at near zero field, then decreases monotonically with increasing field; at the frequencies of $5 \mathrm{MHz}, 10 \mathrm{MHz}, 15 \mathrm{MHz}$ (Fig. 4 (b), (c) and (d)), double peaks (DPs) appeared in negative and positive magnetic fields. Especially at $10 \mathrm{MHz}-15 \mathrm{MHz}$, the equivalent magnetic anisotropic field $H_{\mathrm{k}}$ (indicated in the Fig. 4 (d) of TJA wire increases to the maxima near \pm 2.0 Oe before decreasing monotonically with further increase of external fields, whereas $H_{\mathrm{k}}$ of other states are all much smaller. This suggests that the TJA treatment enables the wire to take on a broader working range for weak magnetic field detection than other conventional annealing method discussed here. The GMI peak is mainly attributed to the dynamic 
magnetization process where the dominating magnetization process varies from domain wall movement at relatively low frequency to the magnetization rotation at relatively high frequency [26]. While the internal configuration of magnetization might be dependent on frequency to some extent, the transformation from SP to DPs feature is determined by the relative contribution between the domain wall motion and magnetic moment rotation processes. At relatively low frequency, the domain wall movement plays a key role in the magnetization process, circumferential permeability decreases with increasing axial magnetic field, and then GMI ratio decreases accordingly [27]. On the contrary, at relatively high frequencies when skin-effect became very strong, the eddy-current action results in increased damping and the domain wall motion gives way to magnetic moment rotation to dominate the magnetization process. As a result, the permeability rapidly increases at first and then decreases as the external field increasing as seen in the GMI profiles [28].

The maximum GMI ratios $\left[\Delta \mathrm{Z} / \mathrm{Z}_{0}\right]_{\max }$ are plotted in Fig. 5 as a function of driving frequency. It is clear that an increasing tendency of $\left[\Delta \mathrm{Z} / \mathrm{Z}_{0}\right]_{\max }$ for above mentioned four states is observed with increasing frequency up to $20 \mathrm{MHz}$, and the values of $\left[\Delta \mathrm{Z} / \mathrm{Z}_{0}\right]_{\max }$ at different frequency measured under positive and negative magnetic fields have good repeatability indicated by small error bars. Notably, the $\left[\Delta Z / Z_{0}\right]_{\max }$ values of TJA wire are $59.21 \%$, $100.75 \%, 102.87 \%$ at $5 \mathrm{MHz}, 10 \mathrm{MHz}, 20 \mathrm{MHz}$, respectively, the peak value is $104.29 \%$ with negative magnetic field decreasing to -2.10 at $15 \mathrm{MHz}$. However, the peak values of as-cast, JA and CVA wires are $23.14 \%$ at $20 \mathrm{MHz}, 56.47 \%$ at $15 \mathrm{MHz}$ and $3.40 \%$ at $20 \mathrm{MHz}$, respectively. Therefore, the TJA wire yields a larger GMI ratio than other annealed wires at the frequency range of $1 \mathrm{MHz}-20 \mathrm{MHz}$; TJA should then be considered as an optimized annealing technique to improve GMI performance of amorphous microwires.

It should be noted that TJA is different from conventional JA: TJA has two stages (one long-time current annealing stage at a relatively lower current density and another short-time 
current annealing stage at a relatively high current density, both cooling in air), while JA has only a single current annealing stage with air cooling. As such, TJA has several advantages on the improvements of GMI effect and soft magnetic properties in comparison with conventional JA. In the TJA process, the intensive circular magnetic field induced by a relatively high current density with short-time annealing can lead to the enlargement of circular magnetic anisotropy domain. On the contrary, long-time current annealing using a relatively high current density, such as JA process, will cause a rapid crystallization of microwires, which could be detrimental to the GMI effect. Therefore, it makes much sense to adopt a long-time current annealing stage as the pretreatment, which can release inner residual stress adequately and regulate atomic arrangement state, followed by a short-time current annealing stage that can adjust the distribution of circular magnetic anisotropy domain induced by the self-generated external circular magnetic field. In addition, TJA can effectively avoid the occurrence of rapid crystallization of microwires, and achieve an ideal modulation of GMI effect and soft magnetic properties.

\subsection{Mechanisms behind the GMI improvement under TJA}

GMI effect is strongly related to the skin effect. Using Maxwell's equations, the expression of impedance $Z$ and skin depth $\delta$ can be directly given by $[29,30]$ :

$$
\left\{\begin{array}{l}
Z=R+j X=\frac{1}{2} R_{D C}(k \alpha) \frac{J_{0}(k \alpha)}{J_{1}(k \alpha)} \\
\delta=\sqrt{\frac{\rho}{\mu_{\varphi} \pi f}}
\end{array}\right.
$$

where $R_{D C}$ is the DC resistance; $R, X$ are the real and imaginary part of $Z$, respectively; $J_{i}(k a)$ are $i$-th-order Bessel function of the first kind; $k=(1+j) a / \delta, j$ is the imaginary unit; $a$ is the radius of the wire; $\rho$ is the electrical resistivity; $f$ is the frequency of driving current; $\mu_{\varphi}$ is the circumferential permeability depending on the magnetization process and the structure of magnetic domains. $\mu_{\varphi}, \delta$, and GMI effect can be changed by modulating inner magnetic domain structure called as either core-shell or bamboo-like structure [31, 32]. 
For TJA wire with enhanced GMI effect, it can be explained by the following mechanisms: (i) stress relief mechanism. During the annealing process, micro-regions are formed by atomic diffusion and stress relief under the action of thermal activation energy. Cobalt atoms have affinity to silicon atoms, and iron atoms to boron atoms, leading to the formation of many RAAMs. Meanwhile, annealing at $80 \mathrm{~mA}$ for $15 \mathrm{~min}$ is the main stress releasing stage, while annealing at $110 \mathrm{~mA}$ for $5 \mathrm{~min}$ is the treatment stage generating strong external circular magnetic field which can dramatically increase circumferential magnetic anisotropic field in the wire, enabling the TJA wire to show the best possible GMI performance according to the Equation (4); (ii) critical rotational magnetization mechanism. RAAMs of suitable size and volume fraction are generated during annealing, which could damp the motion of domain wall for nail-sticked action and eddy-current damping but not seriously decrease the circumferential magnetic anisotropic field without excess crystallization. This consequently increases the amount of magnetic moments rotated to the final equilibrium position by the external field $H_{\mathrm{ex}}$ corresponding to the maximum $\Delta \mathrm{Z} / \mathrm{Z}_{0}$, which yields a remarkable increase in the magnetic permeability and hence GMI effect [33]. Therefore, an appropriate volume fraction of RAAMs in annealed wire is desirable for optimizing GMI effect. On the contrary, after CVA (at $773 \mathrm{~K}$ for $20 \mathrm{~min}$ ), a large amount of hard magnetic nanophases, i.e. CoSi, are precipitated with a volume fraction exceeding $5 \%$. The coercivity increases from $20.5 \mathrm{~A} / \mathrm{m}$ of as-cast wire to $32.6 \mathrm{~A} / \mathrm{m}$ of $\mathrm{CVA}$ wire, the maximum magnetic permeability increases from 0.0077 of as-cast wire to 0.0106 of CVA wire, as shown in Fig. 6. These modified magnetic properties can be accounted for by the effect of MRORs, which lead to an increase of magnetocrystalline anisotropy and a considerable decrease of magnetic moment exchange coupling.

Fig. 7 reveals the dependences of maximum field response sensitivity $\xi_{\max }$ on frequency, ranging from $100 \mathrm{kHz}$ to $20 \mathrm{MHz}$. At initial stage of $100 \mathrm{kHz}-1 \mathrm{MHz}, \xi$ varies inconspicuously 
with frequency. Of particular interest is that the twin-zone Joule annealed (TJA) wire exhibits larger $\xi$ values than the as-cast, JA and CVA wires at the frequency range of $1 \mathrm{MHz}-20 \mathrm{MHz}$. Their $\xi_{\max }$ values are $57.36 \% / \mathrm{Oe}, 164.11 \% / \mathrm{Oe}, 162.59 \% / \mathrm{Oe}$, and the corresponding errors are $3.46 \% / \mathrm{Oe}, 0.75 \% / \mathrm{Oe}, 3.00 \% / \mathrm{Oe}$ at $5 \mathrm{MHz}, 10 \mathrm{MHz}, 20 \mathrm{MHz}$, respectively. The maximum field sensitivity reaches to $181.83 \% / \mathrm{Oe}$ with positive magnetic field increasing to $0.6 \mathrm{Oe}$ at $12 \mathrm{MHz}$. Therein, the sensor applied region (SAR) between $10 \mathrm{MHz}-15 \mathrm{MHz}$ is very significant for sensor application. In general, the annealed wire is required to have significant GMI effect (GMI ratio and higher field response sensitivity exceed 100\% and 160\%/Oe at relatively low frequency, respectively) for magnetic sensor application. Especially, the magnitude of field sensitivity is significant for application at relatively low frequency and low magnetic field, i.e., a small external magnetic field can generate a large impedance change, corresponding to large voltage change. The lower driving frequencies, the easier treatment of noise signal in electronic circuit of magnetic sensor. Comparing with JA and CVA, the TJA wire has significantly higher GMI ratio $\Delta \mathrm{Z} / \mathrm{Z}_{0}$ of $104.29 \%$ at $15 \mathrm{MHz}$ and maximum field sensitivity $\xi_{\max }$ of $181.82 \% / \mathrm{Oe}$ at $12 \mathrm{MHz}$ comparing with previous report of GMI effect (GMI ratio of $50 \%$ at $10 \mathrm{MHz}$ ) for the melt-extracted Co-based microwire [10], making it promising for magnetic sensor application at megahertz frequencies.

The last point deals with the evolution of the circumferential magnetic anisotropy and magnetostriction constant of microwires after CVA, JA and TJA annealings, which is considered of crucial importance for GMI effect optimization [34, 35]. It is known that the magnetostriction coefficient can be expressed as [36-38]:

$$
\lambda_{s}(\sigma)=\lambda_{s, 0}-A \sigma
$$

where $\lambda_{s, 0}$ is the saturation magnetostriction coefficient without tensile stress and $A$ is a small positive coefficient correlative to heat-treatments. $\sigma$ denotes the total of internal and external stresses. As $A$ is way smaller than $\sigma$ by several order of magnitude, it follows that the 
magnetostriction coefficient is mainly dependent on $\sigma$. Due to the stress relief caused by annealing, the negative magneostriction coefficient is then increased, i.e, closer to zero. With the coupling of a closer-to-zero magnetostriction coefficient and smaller residual stress, the magnetoelastic energy then becomes smaller, and hence the GMI properties (GMI ratio and field sensitivity) are improved. In this regard, the stronger reinforcement of GMI effect due to TJA than other techniques could be attributed to a correspondingly stronger stress relief. From the mictrostructure point of view, the RAAMs generated by TJA reinforce the circumferential anisotropy domain by the "nail-stick" action during magnetization [32, 39, 40]. Therefore, TJA results in smaller magnetoelastic energy and more regular magnetic domain structure, thus improving both magnetization process (domain wall motion and moment rotation) and GMI properties. For the single-stage JA wires, it is difficult to obtain a well-defined circumferential anisotropy and suitable RAAMs. For the CVA wires, the generated MRORs take on obviously crystalline characteristics, hence the formation of CoSi plays a critical role in governing the GMI effect. As the direction of magnetic moments for $\mathrm{CoSi}$ is along the axis of wire, they can decrease the volume of circumferential anisotropy domain. In consequence, the CVA wire exhibits a small GMI effect with a larger magnetoanisotropy than as-cast wire and JA wires. Moreover, it is hard to obtain high magnetic inductivity at relatively high frequency, in that most moments in different domains are rotated or driven by the driving force to the final equilibrium position.

\section{Conclusion}

In summarizing, TJA has a profound effect on microstructure and GMI property. Quantitatively, TJA (annealing at $80 \mathrm{~mA}$ for $15 \mathrm{~min}$ and increasing to $110 \mathrm{~mA}$ for $5 \mathrm{~min}$ then air cooling) technique enables the wire to exhibit a maximum GMI ratio $\left[\Delta Z / Z_{0}\right]_{\max }$ of $104.29 \%$ at $15 \mathrm{MHz}$ and the maximum field response sensitivity $\xi_{\max }$ of $181.82 \% / \mathrm{Oe}$ at $12 \mathrm{MHz}$, and also has about twice wider working range $( \pm 2.0 \mathrm{Oe})$ for weak magnetic field detection than 
as-cast and other annealed wires. The enhanced GMI effect for TJA wires is elucidated from the standpoint of microstructural evolution. Specifically, RAAMs with suitable size and volume fraction are found to result in a remarkable increase of magnetic permeability and GMI effect by increasing the amount of magnetic moment rotated to the final equilibrium position by the external field $H_{\mathrm{ex}}$. However, the formation of MRORs and hard magnetic nanophases in CVA wire severely deteriorate the GMI performance. It can therefore be concluded that TJA is an efficient technique to reinforce the GMI ratio and field response sensitivity of wires, making the wires suitable for high-performance sensor applications at megahertz frequencies.

In the future work, the TJA technique can be further optimized with manipulation of relevant annealing parameters, such as current intensity and annealing time. It is also viable to update this technique with cooling medium and/or combination with other special annealing, such as AC \& pulse current annealing. HRTEM and FFT/IFFT transformation will continue to be employed as they can provide more direct evidence to establish a clear structure-property relationship and give us a better understanding of the relevant magnetic characteristics of structural reasons.

\section{Acknowledgements}

J. S. Liu is grateful to Prof. G. Wang, Shanghai University, China, for his warm help with the magnetic measurement of Curie temperature. And J. S. Liu also would like to thank Dr. X. H. Hou, Inner Mongolia University of Technology, China, for his assistance to the preparation of test samples, HRTEM observation and valuable discussions.

\section{References}

[1] M. Vázquez, Physica B 299 (2001) 302-313.

[2] H. Hauser, L. Kraus, P. Ripka, IEEE Trans. Magn. 4 (2001) 28-32.

[3] M.-H. Phan, H.-X. Peng, Prog. Mater. Sci. 53 (2008) 323-420. 
[4] H. Chiriac, T.-A. Óvári, J. Magn. Magn. Mater. 323 (2011) 2929-2940.

[5] H. Chiriac, T.-A Óvári, Prog. Mater. Sci. 40 (1996) 333-407.

[6] H. Wang, D.W. Xing, X.D. Wang, Metall. Mater. Trans. A 42A (2011) 1103-1108.

[7] I. Ogasawara, S. Ueno, IEEE Trans. Magn. 31 (1995) 1219-1223.

[8] H. Chiriac, Mater. Sci. Eng. A 304-306 (2001) 166-171.

[9] P. Ciureanu, I. Khalil, L.G.C. Melo et al., J. Magn. Magn. Mater. 249 (2002) 305-309.

[10] V. Zhukova, A. Zhukov, K. García, et al., Sens. Acta. A-Phys 106 (2003) 225-229.

[11] L.V. Panina, J. Magn. Magn. Mater. 249 (2002) 278-287.

[12] X. Zhou, G. Tu, H. Kunkel, et al., Sens. Acta. A-Phys 125 (2006) 387-392.

[13] A. Zhukov, M. Ipatov, J.M. Blanco, et al., Phys. Status Solidi A 206 (2009) 674-678.

[14] C. Moron, A. Garcia, J. Magn. Magn. Mater. 290 (2005) 1085-1088.

[15] V. Zhukova, A.F. Cobeño, A. Zhukov, et al., J. Non-Cryst. Solids. 287 (2001) 31-36.

[16] K.R. Pirota, L. Kraus, H. Chiriac et al., J. Magn. Magn. Mater. 221 (2000) L243-L247.

[17] J.M. Blanco, A. Zhukov, A.P. Chen, et al., J. Phys. D: Appl. Phys. 34 (2001) L31-L34.

[18] K.J. Jang, C.G. Kim, S.S. Yoon, et al., IEEE Trans. Magn. 35 (1999) 3889-3891.

[19] S.L. He, K.Y. He, Z. Wang, et al., Chin. Phys. Lett. 14 (1997) 464-467.

[20] M.-H. Phan, H.-X. Peng, M. R. Wisnom, et al., Compos. Part A-Appl. S. 37 (2006) 191-196.

[21] J.F. Hu, H.W. Qin, F. Zhang, et al., J. Appl. Phys. 91 (2002) 7418-7420.

[22] J.S. Liu, J.F. Sun, D.W. Xing, et al., Phys. Status Solidi A 208 (2011) 530-534.

[23] M.M.J. Treacy, J.M. Gibson, L. Fan, et al., Rep. Prog. Phys. 68 (2005) 2899-2944.

[24] F.P. Incropera, D.P. DeWitt, Fundamentals of Heat Transfer, John Wiley \& Sons Inc, 1981, p. 295-370.

[25] A. Zhukov, M. Ipatov, J.Gonzalez et al., J. Magn. Magn. Mater. 321 (2009) 822-825.

[26] R. Varga, Y. Kostyk, K. Richter, et al., Phys. Status Solidi A 206 (2009) 608-612. 
[27] R. Valenzuela, Physica B 299 (2001) 280-285.

[28] A. Chizhika, C. Garciaa, A. Zhukova, et al., Physica B 384 (2006) 5-8.

[29] L.V. Panina, K. Mohri, T. Uchiyama, et al., IEEE Trans. Magn. 31 (1995) 1249-1260.

[30] M. Knobel, M.L. Sánchez, C. Gómez-Polo, et al., J. Appl. Phys. 79 (1996) 1646-1654.

[31] N. Usov, A. Antonov, A. Dykhne et al., J. Magn. Magn. Mater. 174 (1997) 127-132.

[32] J.F. Sun, J.S. Liu, D.W. Xing, et al., Phys. Status Solidi A 208 (2011) 910-914.

[33] V. Zhukova, J.M. Blanco, M. Ipatov, et al., Phys. Status Solidi A 206 (2009) 679-682.

[34] A. Zhukov, Adv. Funct. Mater. 16 (2006) 675-680.

[35] M.L. Sartorelli, M. Knobel, J. Schoenmaker, et al., Appl. Phys. Lett. 71 (1997) $2208-2210$

[36] A. Zhukov, V. Zhukova, J.M. Blanco, et al., J. Magn. Magn. Mater. 258-259 (2003) 151-157.

[37] A. Zhukov, K. García, M. Kuzminski, et al., Sens. Transd. Mag. 41 (2004) 174-180.

[38] F. Qin, V.V.Popov, H.-X. Peng, et al., J. Alloys Compd. 509 (2011) 9508-9512.

[39] J.S. Liu, J.F. Sun, D.W. Xing, et al., Mater. Technol. 27 (2012) 142-144.

[40] J.S. Liu, D.Y. Zhang, F.Y. Cao, et al., Phys. Status Solidi A 209 (2012) 984-989. 


\section{Figure Captions}

Fig. 1. Schematic diagrams showing the steps for HRTEM sample preparation.

Fig. 2. X-ray diffraction patterns of as-cast and annealed $\mathrm{Co}_{68.2} \mathrm{Fe}_{4.3} \mathrm{~B}_{15} \mathrm{Si}_{12.5}$ microwires.

Fig. 3. HRTEM images and patterns (including FFT and IFFT micrographs) of Co-based amorphous wires for different states: as-cast (a), JA (b), TJA (c) and CVA (d).

Fig. 4. GMI profiles of of as-cast and annealed wires at different frequencies: $f=100 \mathrm{kHz}(\mathrm{a})$, $f=5 \mathrm{MHz}(\mathrm{b}), f=10 \mathrm{MHz}(\mathrm{c}), f=15 \mathrm{MHz}(\mathrm{d})$. The bidirectional arrows in (d) indicate the positions of equivalent magnetic anisotropy field $H_{\mathrm{k}}$ and $H_{\mathrm{k}}$.

Fig. 5. Frequency dependences of maximum GMI ratio $\left[\Delta \mathrm{Z} / \mathrm{Z}_{0}\right]_{\max }$ for as-cast and annealed Co-based microwires. The inset rectangle indicates the sensor applied region (SAR) of TJA wire, which has the maximum GMI ratio at the frequency ranging from $10 \mathrm{MHz}$ to $15 \mathrm{MHz}$.

Fig. 6. The longitudinal hysteresis loops $(M-H)$ of melt-extracted wire (a), and CVA wire (b). The unidirectional arrows indicate their coercivities that were measured by electromagnetic inductive method (EIM).

Fig. 7. The variation curves of maximum field response sensitivity $\xi_{\max }(\% / \mathrm{Oe})$ at various frequencies. The inset rectangle indicates the sensor applied region (SAR) of TJA wire, which has the best response sensitivity at the frequency range of $10 \mathrm{MHz}-15 \mathrm{MHz}$. 